\title{
Antiphospholipid antibodies: a possible biomarker of disease activity in multiple sclerosis and neuromyelitis optica spectrum disorders
}

\author{
Tatiana Koudriavtseva • Domenico Plantone • \\ Rosaria Renna
}

Received: 21 July 2014/Revised: 11 September 2014/Accepted: 12 September 2014/Published online: 19 September 2014

(c) Springer-Verlag Berlin Heidelberg 2014

\section{Dear Sirs,}

Multiple sclerosis (MS) and neuromyelitis optica spectrum disorders (NMOSD) are chronic inflammatory demyelinating disease of the central nervous system. MS usually begins with a relapsing-remitting course manifesting with relapses followed by recovery. Most patients will enter a secondary progressive phase characterized by steady accumulation of disability. NMOSD is a more severe disorder characterized by optic neuritis, longitudinally extensive myelitis and autoantibody positivity against the water channel aquaporin-4. It has been demonstrated that reactivity for different autoantibodies, including antinuclear and antiphospholipid antibodies (aPL), is more frequent in NMOSD compared to MS [1, 2].

Iong and colleagues [1] have confirmed a significantly higher positive rate for anticardiolipin antibodies (aCL) in NMOSD $(45.7 \%)$ compared to MS (5.6\%). We found a similar aCL positivity $(5 \%)$ in MS patients, although the overall aPL positive rate increased up to $60 \%$ when the reactivity for more aPL was evaluated [3]. Based on the literature data, it appears that the rate of aPL positivity in MS is related to both the number of antibodies used and the severity of the disease stage [3-6]. Indeed, aPL positivity was reported to be higher in secondary progressive than in relapsing-remitting phase $[4,5]$, and to be correlated with the T2-lesion volume at MRI [5]. Moreover, aPL positive relapsing-remitting MS patients developed more severe clinical and MRI disease progression compared to aPL

T. Koudriavtseva $(\varangle) \cdot$ D. Plantone $\cdot$ R. Renna

Multiple Sclerosis Center, Unit of Neurology, Regina Elena

National Cancer Institute, IFO, Via Elio Chianesi 53,

00144 Rome, Italy

e-mail: tatiana.koud@gmail.com; koudriavtseva@ifo.it negative ones over a 3-year follow-up [7]. Nevertheless, the highest rate of aPL positivity (about $80 \%$ ) was found only during MS relapse $[3,4,6]$ correlating with the number of MRI enhancing lesions [6]. Interestingly, aPL positivity significantly decreased in the same patients in remission [3, 4].

Iong's paper [1] has reported a similar trend also in NMOSD: patients positive for both IgG and IgM aCL were older and with a higher level of disability compared to patients positive only for IgG aCL who were, in turn, older than aCL negative patients. Moreover, aCL positivity in NMOSD patients has been associated with greater antithrombin-III activity and D-dimer levels, a product of fibrin degradation, thus confirming the association of aCL with thrombotic processes. To our knowledge, only few studies have evaluated coagulation factors in MS [8-13], generally confirming the activation of the coagulation cascade. However, the presence of thrombotic processes in MS has been well-recognized [14].

Since aPL are found not only in antiphospholipid syndrome (APS), NMOSD and MS, but also in many inflammatory diseases and infections, it is reasonable to suppose that they may be a nonspecific biomarker of underlying universal inflammatory-thrombotic events. Thus, aPL may reflect the intensity of these pathological processes i.e. the disease severity/activity rather than its specificity. In fact, another study, based on patients' positivity for anti- $\beta 2$ glycoprotein-I, has classified the different types of MRI cerebral lesions in: typical inflammatory, typical vascular and the most common atypical lesions, proposed by the authors as a frontier syndrome between APS and MS [15]. This could also mean that aPL are prevalently involved in the clearance of damaged molecular and cellular components containing phospholipids rather than triggering the disease. Therefore, the higher aPL reactivity rate in 
NMOSD compared to MS could reflect its more severe character, as the contradictory results of aPL positivity reported in MS patients [3-6, 16] may be due to the heterogeneity of the disease.

Certainly, there is a pathological continuum involving inflammation and coagulation, two essential arms of nonspecific innate immunity closely correlated with the specific adaptive immunity. Further extensive and detailed studies on the coagulation system in NMOSD and MS are needed to better understand its pathogenic role and to evaluate the opportunity of anticoagulant treatment in inflammatory-demyelinating disorders.

Conflicts of interest No conflict of interest or financial interest is reported. T. Koudriavtseva reports consulting fees from Bayer Schering, and Institutional grant from Merck Serono, Biogen Idec, Novartis, Bayer Schering outside the submitted work. Other authors have nothing to declare.

Ethical standard All human studies must state that they have been approved by the appropriate ethics committee and have therefore been performed in accordance with the ethical standards laid down in the 1964 Declaration of Helsinki.

\section{References}

1. Iong Y, He Y, Zheng Y, Chen M, Zhang B, Gao C (2013) Serum anticardiolipin antibodies in patients with neuromyelitis optica spectrum disorder. J Neurol 260(12):3150-3157

2. Wu L, Huang D, Yang Y, Wu W (2014) Combined screening for serum anti-nuclear and anti-aquaporin- 4 antibodies improves diagnostic accuracy for distinguishing neuromyelitis optica from multiple sclerosis. Eur Neurol 72(1-2):103-108

3. Koudriavtseva T, D'Agosto G, Mandoj C, Sperduti I, CordialiFei P (2014) High frequency of antiphospholipid antibodies in relapse of multiple sclerosis: a possible indicator of inflammatory-thrombotic processes. Neurol Sci. doi:10.1007/s10072-0141823-4

4. Spadaro M, Amendolea MA, Mazzucconi MG, Fantozzi R, Di Lello R, Zangari P, Masala G (1999) Autoimmunity in multiple sclerosis: study of a wide spectrum of autoantibodies. Mult Scler 5(2): $121-125$

5. Garg N, Zivadinov R, Ramanathan M, Vasiliu I, Locke J, Watts K, Lema J, Rajeswary J, Munschauer FE, Ambrus J Jr, Weinstock-Guttman B (2007) Clinical and MRI correlates of autoreactive antibodies in multiple sclerosis patients. J Neuroimmunol 187(1-2):159-165

6. Bidot CJ, Horstman LL, Jy W, Jimenez JJ, Bidot C Jr, Ahn YS, Alexander JS, Gonzalez-Toledo E, Kelley RE, Minagar A (2007) Clinical and neuroimaging correlates of antiphospholipid antibodies in multiple sclerosis: a preliminary study. BMC Neurol $7: 36$

7. Zivadinov R, Ramanathan M, Ambrus J, Hussein S, Ramasamy DP, Dwyer MG, Bergsland N, Minagar A, Weinstock-Guttman B (2012) Anti-phospholipid antibodies are associated with response to interferon-beta1a treatment in MS: results from a 3-year longitudinal study. Neurol Res 34(8):761-769. doi:10.1179/ 1743132812Y.0000000076

8. Brunetti A, Ricchieri GL, Patrassi GM, Girolami A, Tavolato B (1981) Rheological and fibrinolytic findings in multiple sclerosis. J Neurol Neurosurg Psychiatry 44(4):340-343

9. Kirichuk VF, Streknev AG (2003) The role of hemostasis system in the pathogenesis and course of multiple sclerosis. Zh Nevrol Psikhiatr Im S S Korsakova (Spec No 2):34-38

10. Aksungar FB, Topkaya AE, Yildiz Z, Sahin S, Turk U (2008) Coagulation status and biochemical and inflammatory markers in multiple sclerosis. J Clin Neurosci 15(4):393-397. doi:10.1016/j. jocn.2007.02.090

11. Campos-de-Magalhães $\mathrm{M}$, de Almeida AJ, Papaiz-Alvarenga RM, Gadelha T, Morais-de-Sá CA, Alves-Leon SV (2009) Normal plasma antithrombin activity in patients with relapsingremitting and secondary progressive multiple sclerosis. Clin Neurol Neurosurg 111(5):407-411. doi:10.1016/j.clineuro.2008. 11.016

12. Ingram G, Hakobyan S, Hirst CL, Harris CL, Loveless S, Mitchell JP, Pickersgill TP, Robertson NP, Morgan BP (2012) Systemic complement profiling in multiple sclerosis as a biomarker of disease state. Mult Scler 18(10):1401-1411

13. Festoff BW, Li C, Woodhams B, Lynch S (2012) Soluble thrombomodulin levels in plasma of multiple sclerosis patients and their implication. J Neurol Sci 323(1-2):61-65. doi:10.1016/ j.jns.2012.08.008

14. Koudriavtseva T (2014) Thrombotic processes in multiple sclerosis as manifestation of innate immune activation. Front Neurol 5:119. doi:10.3389/fneur.2014.00119

15. Renaud M, Aupy J, Uring-Lambert B, Chanson JB, Collongues N, Blanc F, De Sèze J (2014) Isolated anti- $\beta 2$-glycoprotein I antibodies in neurology: a frontier syndrome between multiple sclerosis and antiphospholipid syndrome? Eur J Neurol 21(6):901-906. doi:10.1111/ene.12408

16. Sastre-Garriga Reverter JC, Font J, Tintoré M, Espinosa G, Montalban X (2001) Anticardiolipin antibodies are not a useful screening tool in a nonselected large group of patients with multiple sclerosis. Ann Neurol 49(3):408-411 